\title{
A New Strategy for the Synthesis of Size-controlled L1 0 FePt Nanoparticles on Nanotube Substrate
}

\author{
Reza Moradi $^{1} *$, Seyed Ali Sebt ${ }^{2}$, Hadi Arabi ${ }^{3}$ and Arsalan Beigzadeh ${ }^{1}$ \\ ${ }^{\text {I} D e p a r t m e n t ~ o f ~ P h y s i c s, ~ Q a e m s h a h r ~ B r a n c h, ~ I s l a m i c ~ A z a d ~ U n i v e r s i t y, ~ Q a e m s h a h r, ~ I r a n ; ~}{ }^{2}$ Department of Physics, \\ Science and Research Branch, Islamic Azad University, P.O. Box 14665-678, Tehran, Iran; ${ }^{3}$ Department of Physics, \\ Faculty of Science, Ferdowsi University of Mashhad, Mashhad, Iran
}

\begin{abstract}
In this paper, a CVD method is used for the synthesis of multi-wall carbon nanotubes (MWCNT) which have been purified and functionalized. The approach consists of thermal oxidation and subsequent chemical oxidation. According to TEM images, the CNTs have a diameter about 20-30 nm. We synthesized the FePt nanoparticles on the surface of the functionalized carbon nanotubes through a polyol process. The synthesized FePt nanoparticles have the chemicallydisordered face-centered cubic (fcc) structure with superparamagnetic behavior and with a size of about $2.5 \mathrm{~nm}$. To achieve phase transition from fcc to ordered structure (fct-L1 $1_{0}$ phase), high-temperature annealing under a reducing atmosphere $\left(90 \% \mathrm{Ar}+10 \% \mathrm{H}_{2}\right)$ is required. The CNTs as a substrate prevent the aggregation of particles during thermal treatment. The FePt nanoparticles after phase transition have ferromagnetic behavior. Furthermore, they have finite size with an average about $5.6 \mathrm{~nm}$ and their coercivity reaches to $5.1 \mathrm{KOe}$ at $700^{\circ} \mathrm{C}$. We characterized the structure, composition and magnetic properties of FePt/CNT by X-ray diffraction (XRD), transmission electron microscopy (TEM), field emission scanning electron microscope (FE-SEM), Fourier transform infrared spectroscopy (FT-IR) and vibrating sample magnetometer (VSM).
\end{abstract}

Keywords: Carbon nanotubes, chemically-disordered structure, fcc and fct- $\mathrm{L}_{0}$ structure, FePt nanoparticles, ferromagnetic, superparamagnetic.

\section{INTRODUCTION}

$\mathrm{L} 1_{0} \mathrm{FePt}$ nanoparticles with face-centered tetragonal (fct) structure have very high magnetocrystalline anisotropy $\left(\mathrm{k}_{\mathrm{u}}=7 \times 10^{7} \mathrm{erg} / \mathrm{cm}^{3}\right)$ as compared to other nanoparticles. So, they have very high coercivity which makes them suitable for using in different applications such as ultrahigh density magnetic recording media [1,2], permanent magnets, magnetic sensors and drug delivery in biomedical [3-7]. For the synthesis of FePt nanoparticles with uniform size, chemical methods are suitable. But, the as-synthesized nanoparticles have disordered A1 phase with face-centered cubic (fcc) structure that they shows superparamagnetic behavior at room temperature because of their small anisotropy $[8,9]$. Therefore, to transform $\mathrm{FePt}$ nanoparticles from the A1 phase to the $\mathrm{L}_{0}$ phase, a thermal treatment is required. Since nanoparticles aggregate at high temperature, their size increases and making them inappropriate [10].

In recent years, different processes have been developed for preventing the aggregation of FePt nanoparticles, such as: decreasing of phase transition temperature using the additive metal like $\mathrm{Au}, \mathrm{Ag}$ and $\mathrm{B}_{2} \mathrm{O}_{3}$ [11-13], the salt method $(\mathrm{NaCl})[14,15]$, direct synthesis method [16], rapid thermal annealing [17]. Also, Core/shell structure is an interesting

\footnotetext{
*Address correspondence to this author at the Department of Physics, Qaemshahr Branch, Islamic Azad University, Qaemshahr, Iran; Tel: +98 938 4521722(mobile); Fax: +98 151 2277733;

E-mail: reza.moradi.58@gmail.com
}

approach to prevent coalescence of FePt nanoparticles where the magnetic cores are coated with non-magnetic oxide shells, $\mathrm{SiO}_{2}$ [18], and $\mathrm{MgO}$ or magnetic shell $\mathrm{Fe}_{3} \mathrm{O}_{4}$ and $\mathrm{CoFe}_{2} \mathrm{O}_{4}$ [18-20]. But the results after annealing denote that in these methods, the coercivity decreases significantly. To solve this problem, we use an appropriate substrate for magnetic nanoparticles during the annealing process.

Carbon nanotubes have received extensive attention because of their high electrical, optical and mechanical properties and high thermal stability [21, 22]. Their use is not restricted to just as an adequate substrate to prepare monosize nanoparticles, but they can also be used in high temperature annealing for prevention of particles aggregation from each other. In this paper, has been suggested the coating of the FePt nanoparticles on the CNTs surface and then annealing them at high temperature to phase transition from A1 to fct-L $1_{0}$ phase.

Since these synthesized nanotubes have no ability to absorb Fe or Pt atoms for growth of FePt nanoparticles. Therefore, the nanotubes are functionalized with carboxyl $(-\mathrm{COOH})$ functional groups. The sites of this functional group are an appropriate place for growth of nanoparticles in the reaction media. For functionalization of CNTs, they should be first oxidated at high temperature and then oxidized at the mixture $\mathrm{HCl}, \mathrm{HNO}_{3}$ and $\mathrm{H}_{2} \mathrm{SO}_{4}[23,24]$. $\mathrm{FePt}$ nanoparticles are prepared on carbon nanotubes in the presence of $\mathrm{Pt}(\mathrm{acac})_{2}$ and $\mathrm{Fe}(\mathrm{acac})_{3}$ and 1,2 hexadeconediol as the reduction agent. 
Nanotubes morphology, their coating percent with $\mathrm{FePt}$ nanoparticles in synthesis process, aggregation and changing of their structure after annealing have been studied by XRD, FT-IR, SEM, TEM, EDX and VSM.

\section{EXPERIMENTAL}

\subsection{Synthesis of Functionalized MWCNTs}

In this paper, MWCNTs were prepared through Catalyst Vapor Deposition (CVD) method in tube furnace at $650^{\circ} \mathrm{C}$ using acetylene $\left(\mathrm{C}_{2} \mathrm{H}_{2}\right)$ as carbon precursor and $\mathrm{MgO}$ nanoparticles as catalyst for CNTs growth. To synthesize of CNTs, the flow of acetylene $\left(\mathrm{C}_{2} \mathrm{H}_{2}\right)$ and $\mathrm{Ar}$ gases was passed through the furnace with rates of about $10 \mathrm{sccm}$ and $150 \mathrm{sccm}$, respectively. Then, $\mathrm{MgO}$ nanoparticles were dispersed on quartz glass and inserted into the furnace. After $20 \mathrm{~min}$, quartz glass was removed from furnace with black powder on its surface. Since the resulted products were a mixture of nanotubes, catalyst nanoparticle, florin, amorphous carbon and graphite. They had to be purified and functionalized for growth of $\mathrm{FePt}$ nanoparticles on the surface of CNTs.

Fist, the resulted products were heated under $\mathrm{O}_{2}$ atmosphere at $500^{\circ} \mathrm{C}$ for $90 \mathrm{~min}$. Then, the remaining products were dispersed in the mixture of $\mathrm{HCl}$ and $\mathrm{HNO}_{3}(3 \mathrm{M})$ with ratio $1: 1$ and ultrasound for $10 \mathrm{~min}$. Then, they were stirred at $60^{\circ} \mathrm{C}$ for $2 \mathrm{~h}$. Nevertheless, catalyst nanoparticles and carbon impurities dissolved in the acid solution.

At last, CNTs were immersed in a mixture of $\mathrm{HNO}_{3}$ and $\mathrm{H}_{2} \mathrm{SO}_{4}(3 \mathrm{M})$ with the ratio of $1: 3$ for the functionalization of the CNTs surface with carboxylic functional group [25]. The resulted solution was stirred $(1600 \mathrm{rpm})$ at $90^{\circ} \mathrm{C}$ for $2-4$ hours. Oxidized nanotubes were washed several times with distilled water until the $\mathrm{pH} 6$ was reached and then were dried at $150^{\circ} \mathrm{C}$.

\subsection{Synthesis of FePt/CNT Nanocomposites}

For the growth of FePt nanoparticles on oxidized CNTs, a polyol method was used [1]. Since the oxidized CNTs were dispersed in $40 \mathrm{~mL}$ phenyl ether and ultrasound was performed for $10 \mathrm{~min}$. $\mathrm{Pt}(\mathrm{acac})_{2}(0.5 \mathrm{mmol})$ and $\mathrm{Fe}(\mathrm{acac})_{3}$
(0.5 mmol) salts and also 1,2 hexadeconediol as reducing agent were mixed in above solution. After flowing $\mathrm{N}_{2}$ gas for $10 \mathrm{~min}$ under stirring $(1000 \mathrm{rpm})$, the solution temperature was increased to $100^{\circ} \mathrm{C}$ during $20 \mathrm{~min}$ for starting the reduction of $\mathrm{Fe}$ and $\mathrm{Pt}$ salts and nucleation. During $90 \mathrm{~min}$, the temperature of solution was raised to the boiling point of phenyl ether $\left(259^{\circ} \mathrm{C}\right)$ and was kept at this temperature for 30 min. Finally, the heat source was removed and the solution was left to cool down to room temperature.

$40 \mathrm{ml}$ ethanol was added to purify the product. Then the black product was precipitated and separated via centrifugation $(8000 \mathrm{rpm}, 10 \mathrm{~min})$. The ethanol and impurities were separated, and the discarded black product was dispersed in hexane in the presence of oleic acid and oleyl amine. Centrifugation $(8000 \mathrm{rpm}, 10 \mathrm{~min})$ was performed again to remove any un-dispersed residue.

\subsection{Characterization of Nanocomposites}

Synthesized CNTs were studied before and after purification using FE-SEM (HITACHI S-4160) to determine the purity of nanotubes and their diameter and size. To study the functionalization of nanotubes, FT-IR spectrum (Thermo Nicolet system) was used. The size and shape of the nanoparticles and nanotubes were specified by TEM analysis using a LEO system (9120AB, $120 \mathrm{kV})$. The Fe and Pt elemental analysis of the samples was performed by EDX at 17 $\mathrm{kV}$ using Philips XL30. To determine the structure properties of nanoparticles, the XRD measurement was performed using an X'Pert MPD Philips system with $\mathrm{Cu}-\mathrm{K} \alpha$ radiation $\left(\lambda=1.54 \mathrm{~A}^{\circ}\right)$. The magnetization of FePt samples was measured using a VSM (Lake-Shore model 7400) with the maximum field of up to $20 \mathrm{kOe}$. The nanocomposite annealing was carried in tube furnace under reducing atmosphere $(\% 90$ $\mathrm{Ar}+\% 10 \mathrm{H}_{2}$ ) at $600^{\circ} \mathrm{C}$ for $2 \mathrm{~h}$.

\section{RESULT AND DISCUSSION}

FE-SEM images of CNTs after synthesis and after thermal oxidation have been shown in Fig. (1). Fig. (1a) shows the synthesized CNTs by CVD method. As illustrated in this figure, the CNTs have a diameter between 20-30 nm and
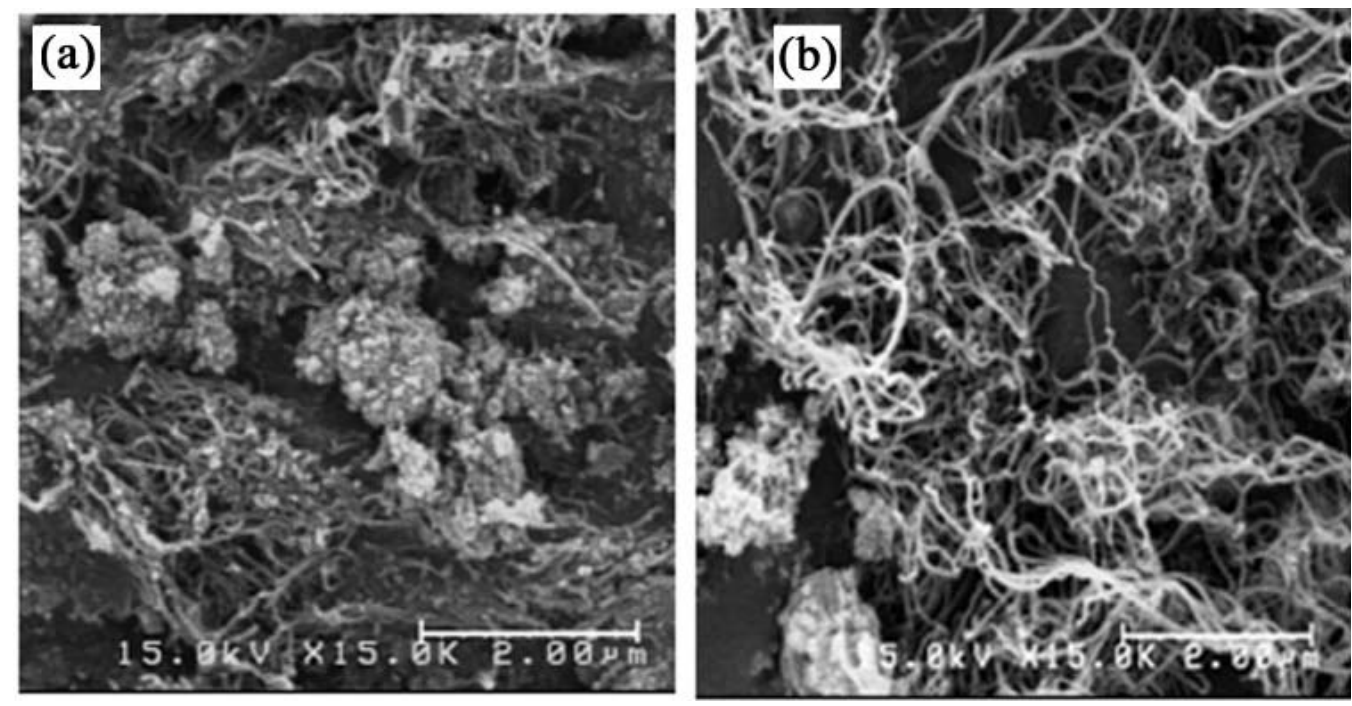

Fig. (1). FE-SEM images of carbon nanotubes and they purification steps: (a) as-synthesis, (b) after thermal oxidation. 
have many impurities. Image of CNTs after thermal oxidation at $500^{\circ} \mathrm{C}$ shown in Fig. (1b), reports the complete removal of amorphous carbon and graphite. They are oxidized quickly and change into $\mathrm{CO}$ and $\mathrm{CO}_{2}$ gas and exit from product. They have free bonds from all sides. But, the CNTs have free bonds only at their two ends, so their oxidation rate is slower and they remain in media.

The XRD patterns of CNTs after oxidation and purification are shown in Fig. (2). According to patterns (2a), there are $\mathrm{MgO}$ peaks with CNTs after oxidation. The catalyst particles peaks are removed completely after purification and only CNTs peaks are observed in XRD pattern (2b). So, the acids oxidation process can dissolve catalyst particles and purified ones.

Fig. (3) shows TEM images of CNTs after thermal oxidation and functionalization. As illustrated in Fig. (3a), there are a lot of $\mathrm{MgO}$ nanoparticles with CNTs. But after functionalization (Fig. 3b), the length of CNTs becomes shorter than before and are removed all catalyst nanoparticles in the final product. Therefore, $\mathrm{HCl}$ and $\mathrm{HNO}_{3}$ can remove all impurities and catalyst particles. According to the figure, the CNTs have an external diameter of about 20-30 nm.
To investigate CNTs functionalization, FT-IR spectrum and dispersion states are used. Fig. (4) shows FT-IR of CNTs before and after functionalization. There are no peaks relating to carboxylic functional groups in Fig. (4a). Hence, they are not functionalized. Fig. (4b and c) show spectrum of CNTs that are functionalized for 2 and 4 hours, respectively. The peak at about $1630 \mathrm{~cm}^{-1}$ relates to $\mathrm{C}=\mathrm{O}$ bonds and the broad peak at $3423 \mathrm{~cm}^{-1}$ relates to $\mathrm{O}-\mathrm{H}$ bonds. So, there are carboxylic functional groups on the surface of CNTs.

According to FT-IR analysis, a lot of active sites on the CNTs surface are made that are useful for nucleation and FePt nanoparticles growth. The peaks intensity of carboxylic group increases with growth of the functionlization time, which shows great number of active sites on the surface of CNTs.

Fig. (5) shows the dispersed states of CNTs samples in aqueous media, at the moment of dispersion and approximately $24 \mathrm{~h}$ and 10 days later. After a surface suffers oxidation, chemical elements are adsorbed and form functional groups. These groups are positively or negatively charged. In this case, carboxylic groups are inserted on the CNTs sur-

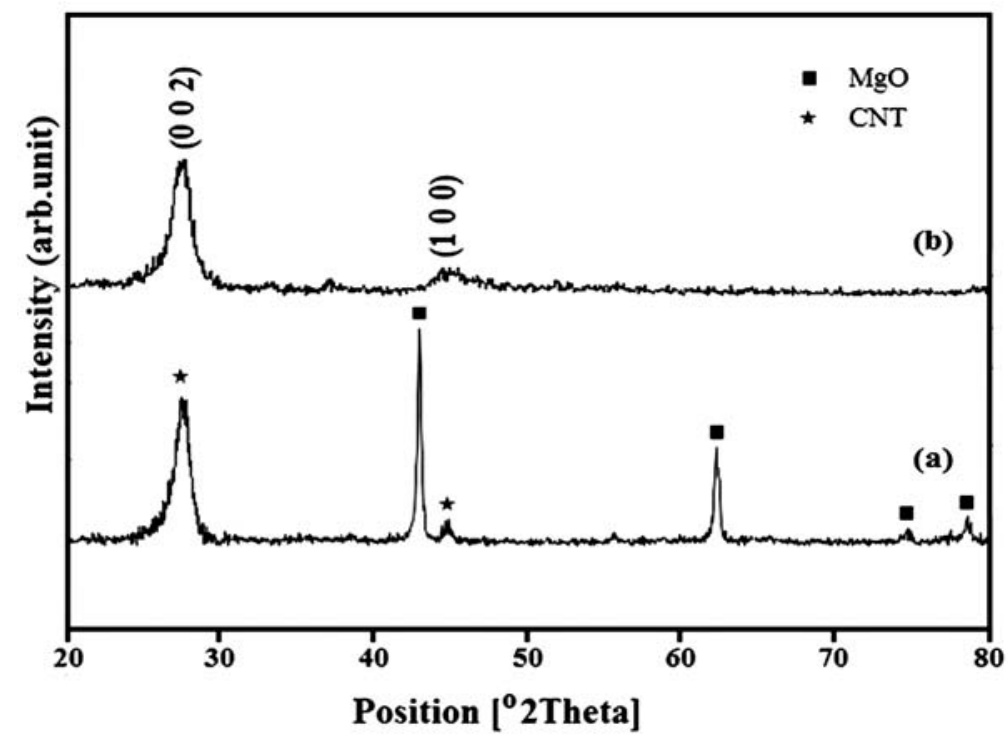

Fig. (2). XRD patterns of CNTs samples after: (a) thermal oxidation and (b) purification.
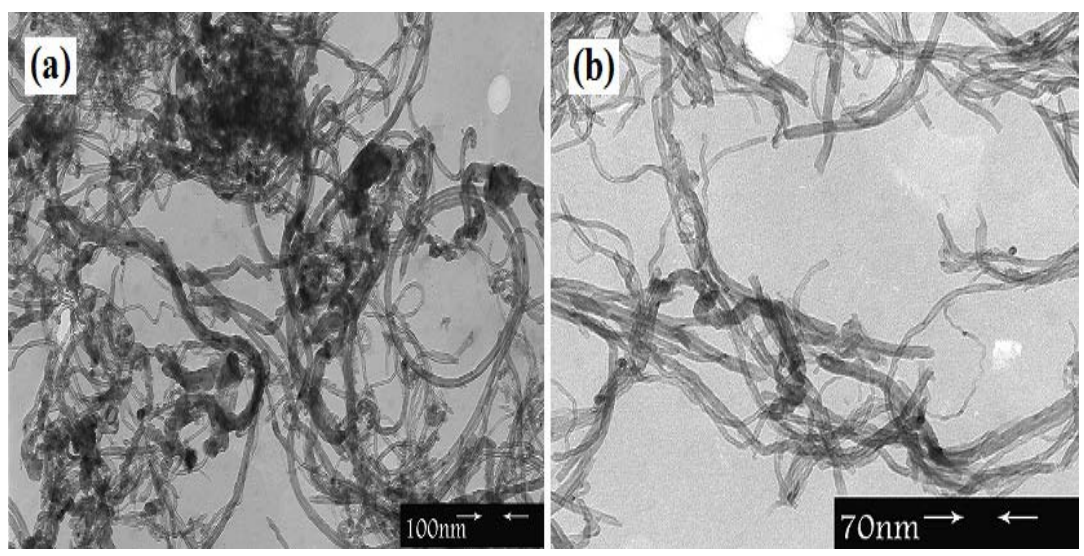

Fig. (3). TEM images of CNTs after: (a) thermal oxidation and (b) functionalization. 


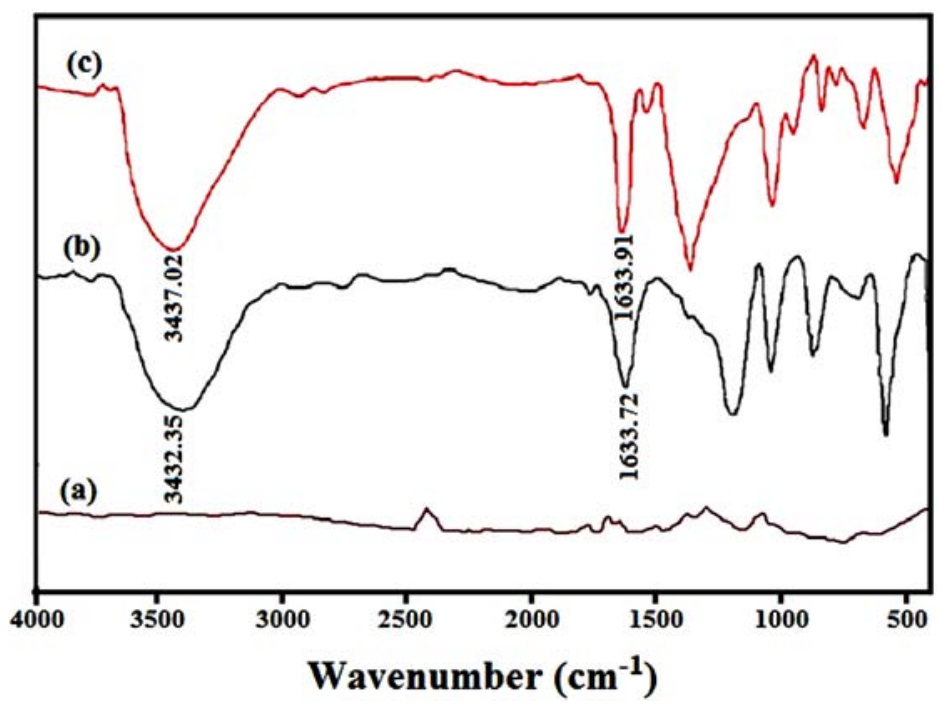

Fig. (4). FT-IR spectrum of CNTs: (a) before functionalization, (b) after functionalization for $2 \mathrm{~h}$ and (c) after functionalization for $4 \mathrm{~h}$.

face, these groups are equally charged. The presence of equal charged particles on the surface of CNTs enables the CNTs to fend from each other, keeping the solution dispersed. Evaluating the precipitation, it is possible to estimate the amount of functional groups adsorbed on the surface of CNTs. Results show a good dispersion of CNTs in an aqueous media, indicating that mostly equally charged hydrocarbons are inserted on the surface of CNTs. Dispersion analysis photographs indicate that the suspension stability of the pristine CNTs was poor as they easily sediment. This sedimentation could be due to the agglomeration of CNTs and lack of hydrogen bonding. But with increasing in functionalization time, the CNTs showed significantly better stability. As the functionalized CNTs for 4 hours presented the best stability, concluding a higher percentage of functional groups absorbed.

Fig. (6A) shows TEM images of synthesized FePt/CNTs nanocomposite which are functinalized for 2 and $4 \mathrm{~h}$, respectively. The black spots on CNTs are according to FePt nanoparticles which grow on its surface. According to Fig. (6Aa and b), the coating of CNTs surfaces improves with increasing in functionalization time and also increases uniformity of functionalized sites on the surface of CNTs. It is necessary to mention that in both, the average size of nanoparticles remains the same as about $2.5 \mathrm{~nm}$.

The histograms of nanoparticles size have been shown in Fig. (6Ba and b). The average size of FePt nanoparticles equals to $2.47 \mathrm{~nm}$ and $2.54 \mathrm{~nm}$, respectively. Their size distribution is narrow even without surfactant and their size is in the range of $1.5-3 \mathrm{~nm}$ with relative standard deviation $(\sigma /<\mathrm{d}>) 0.16$ and 0.21 , respectively.

Fig. (6C) shows the elemental analysis of FePt/CNTs nanocomposite. According to this result, one can deduce the chemical composition of $\mathrm{Fe}_{51} \mathrm{Pt}_{49}$ for the nanocomposite. A stoichiometry close to this one is suitable for forming $\mathrm{L}_{0}$ structure after heat treatment.

Fig. (7) shows XRD patterns of as-synthesized FePt/CNTs nanocomposites which were annealed at $600^{\circ} \mathrm{C}$ and $700^{\circ} \mathrm{C}$ for $2 \mathrm{~h}$ under a reducing atmosphere $(90 \% \mathrm{Ar}+$ $10 \% \mathrm{H}_{2}$ ). According to Fig. (7a), the synthesized nanocom-

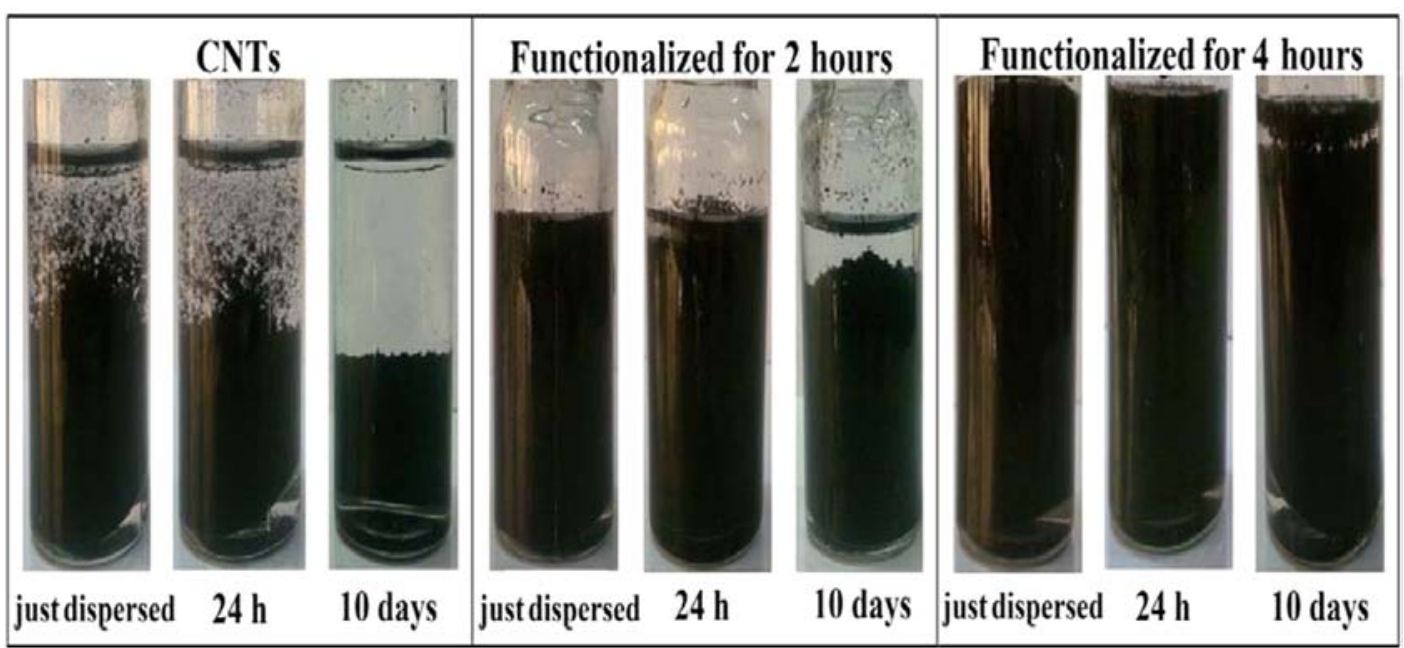

Fig. (5). Dispersion in aqueous media of pristine CNT and functionalized CNTs for 2 and 4 hours: just dispersed; $24 \mathrm{~h}$ and 10 days after. 
(A)
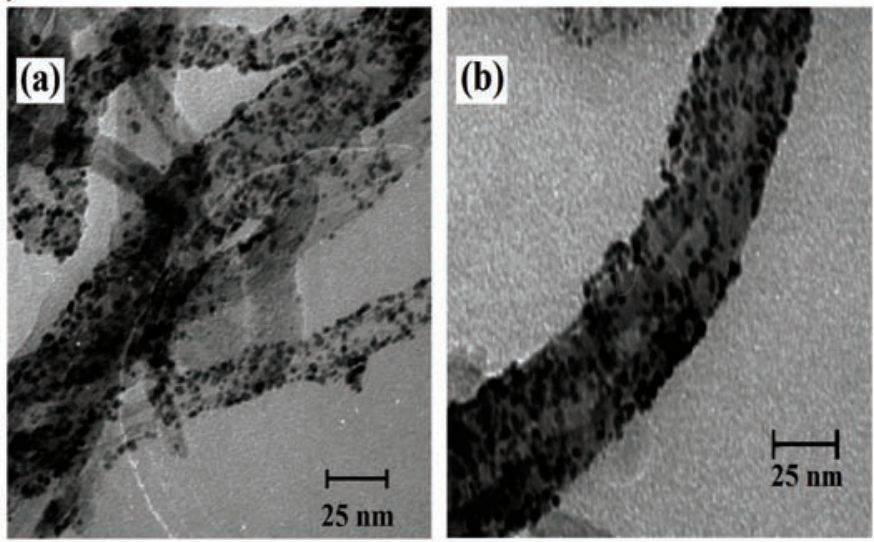

(B)

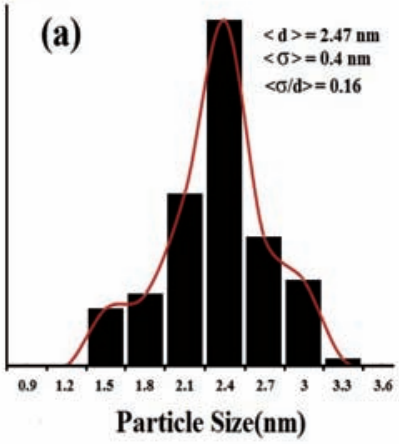

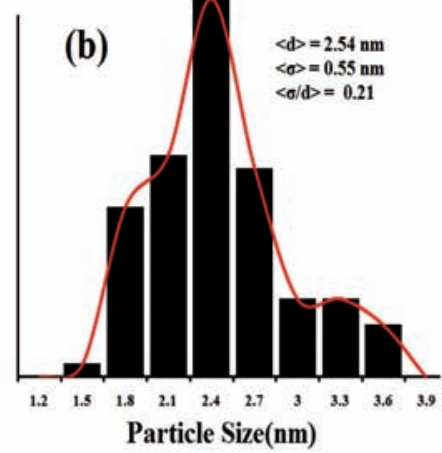

(C)

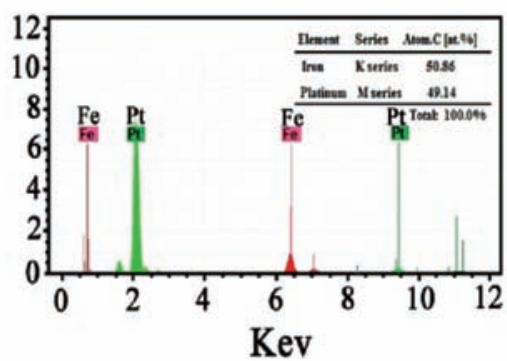

Fig. (6). A) TEM images of FePt nanoparticles on the surfaces of CNTs after functionalization: (a) for $2 \mathrm{~h}$ and (b) for $4 \mathrm{~h}$. B) Histogram of FePt nanoparticles on the surfaces of CNTs after functionalization: (a) for $2 \mathrm{~h}$ and (b) for 4h. Fig. 6C shows EDX image of the as-synthesized fcc FePt nanoparticles.

posites have $\left(\begin{array}{lll}1 & 1 & 1\end{array}\right)$ at $2 \theta=40.23,\left(\begin{array}{lll}2 & 0 & 0\end{array}\right)$ at $2 \theta=47.4$ and (2 20 ) at $2 \theta=67.8$ broad peaks that show the chemically disordered fcc structure and are related to FePt nanoparticles. The average size of FePt particles by Scherrer' equation $\left(D=\frac{k \lambda}{\beta \cos \theta}\right)$ is calculated about $2.9 \mathrm{~nm}$. Fig. (7b and c) show XRD pattern of FePt/CNTs nanocomposite that were annealed at $600^{\circ} \mathrm{C}$ and $700^{\circ} \mathrm{C}$ for $2 \mathrm{~h}$, respectively.

The appearance of ( $\left.\begin{array}{lll}1 & 1 & 0\end{array}\right),\left(\begin{array}{lll}0 & 0 & 1\end{array}\right)$ and $\left(\begin{array}{lll}1 & 1 & 2\end{array}\right)$ peaks and disunion of $\left(\begin{array}{lll}2 & 0 & 0\end{array}\right)$ and $\left(\begin{array}{lll}0 & 0 & 2\end{array}\right)$ peaks from together bode phase start transition from the chemically disordered fcc structure to chemically ordered fct-L $1_{0}$ structure. The difference in lattice constants in direction of $\mathbf{a}$ and $\mathbf{c}$ axis causes separation in location of $\left(\begin{array}{lll}2 & 0 & 0\end{array}\right)$ and $\left(\begin{array}{lll}0 & 0 & 2\end{array}\right)$ peaks. The intensity of diffracted beams is different because of separating in $\mathrm{Fe}$ and Pt crystal planes and variation in electrons density in different planes. Since, the (1 110$),\left(\begin{array}{lll}0 & 0 & 1\end{array}\right),\left(\begin{array}{lll}2 & 0 & 1\end{array}\right)$ and $\left(\begin{array}{lll}1 & 1 & 2\end{array}\right)$ peaks appear in XRD pattern after annealing.

The lattice constant at fcc structure (a) is calculated with location of ( $\left.\begin{array}{lll}1 & 1 & 1\end{array}\right)$ peak in Fig. (7a) that is at $2 \theta=40.32$ and $\mathrm{a}=3.87 \mathrm{~A}^{\circ}$. But, the lattice constants of fct- $\mathrm{L}_{0}$ structure (a and c) for samples which annealed in $600^{\circ} \mathrm{C}$ and $700^{\circ} \mathrm{C}$ are calculated with location of $\left(\begin{array}{lll}1 & 1 & 0\end{array}\right)$ and $\left(\begin{array}{lll}0 & 0 & 1\end{array}\right)$ peaks in Fig. (7b and 7c), respectively and their values are presented at Table 1.
For determination of volume fraction of fct structure, the ordering parameter $S$ is measured which is given by [26]:

$$
\mathrm{S}^{2} \equiv \frac{1-\left(\frac{\mathrm{c}}{\mathrm{a}}\right)}{1-\left(\frac{\mathrm{c}}{\mathrm{a}}\right)_{*}}
$$

That $\left(\frac{c}{a}\right)_{*}$ is the theoretical axis ratio for the chemically ordered fct phase and is equal to 0.96 [27], and $\left(\frac{c}{a}\right)$ is the experimental axis ratio for partially ordered phase. $S=1$ which means that the FePt nanoparticles are fully ordered in the fct phase. The values of $\mathrm{S}$ for FePt nanoparticles that annealed in $600^{\circ} \mathrm{C}$ and $700^{\circ} \mathrm{C}$ are presented in Table 1. Their values are very close to 1 . It means that most FePt nanoparticles have transformed to the chemically ordered fct phase after annealing in temperatures higher than $600^{\circ} \mathrm{C}$ for $2 h$

TEM images of FePt/CNTs nanocomposites that annealed at $600^{\circ} \mathrm{C}$ and $700^{\circ} \mathrm{C}$ for $2 \mathrm{~h}$ have been shown in Fig. (8). Annealing at temperature higher than $600^{\circ} \mathrm{C}$ causes limited coalescence and agglomeration of FePt on the surface of CNTs.

As shown in Fig. (8c and 8d), the nanoparticles size after annealing at $600^{\circ} \mathrm{C}$ and $700^{\circ} \mathrm{C}$ becomes about $3.47 \mathrm{~nm}$ and $5.64 \mathrm{~nm}$, respectively. 


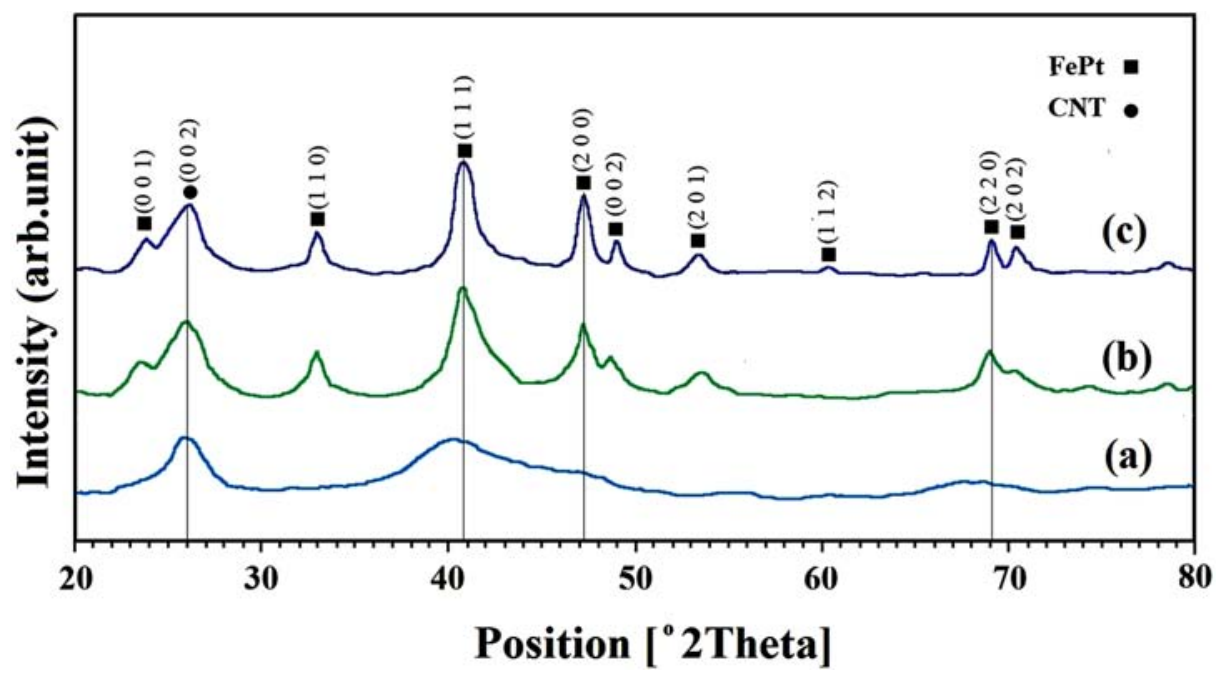

Fig. (7). XRD patterns of FePt/CNT samples: (a) as-synthesis, (b) and (c) after annealing in $600^{\circ} \mathrm{C}$ and $700^{\circ} \mathrm{C}$ for $2 \mathrm{~h}$.

Table 1. Values of lattice constant, ordering parameter and particles size from XRD pattern.

\begin{tabular}{|c|c|c|c|c|c|c|}
\hline Sample & Structure & $\begin{array}{c}\mathbf{a}\left(\mathrm{A}^{\circ}\right) \\
\text { (lattice constant) }\end{array}$ & $\begin{array}{c}\text { c }\left(\mathbf{A}^{\circ}\right) \\
\text { (lattice constant) }\end{array}$ & $\frac{\mathrm{c}}{\mathrm{a}}$ & $\begin{array}{c}\mathbf{S} \\
\text { (ordering parameter) }\end{array}$ & $\begin{array}{c}\text { d }(\mathbf{n m}) \\
(\text { particles size })\end{array}$ \\
\hline as-synthesis & Fcc & 3.87 & - & - & - & 2.9 \\
\hline annealed at $600^{\circ} \mathrm{C}$ & Fct & 3.856 & 3.80 & 0.985 & 0.64 & 3.9 \\
\hline annealed at $700^{\circ} \mathrm{C}$ & Fct & 3.845 & 3.72 & 0.967 & 0.91 & 5.8 \\
\hline
\end{tabular}
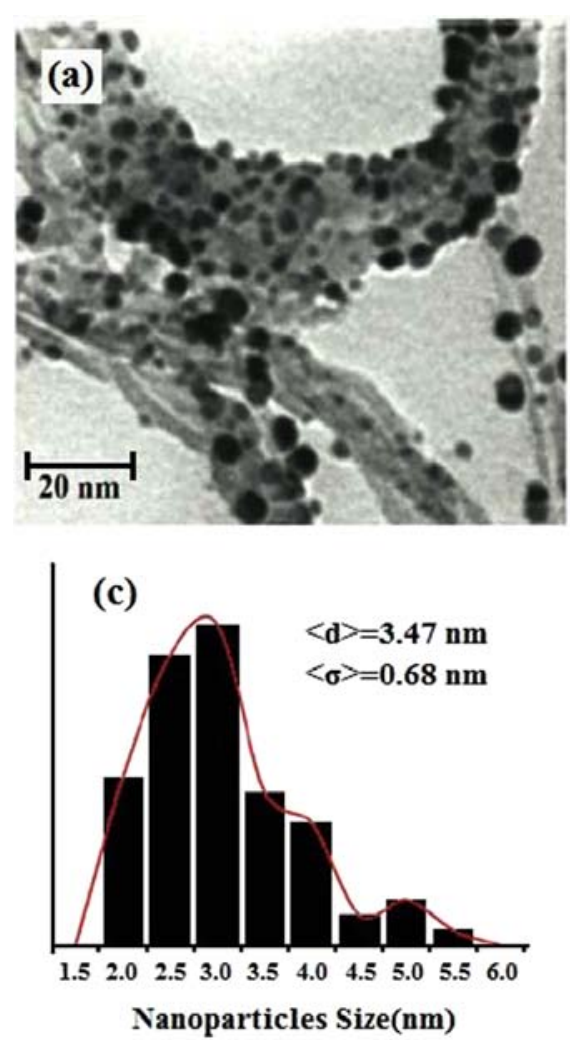
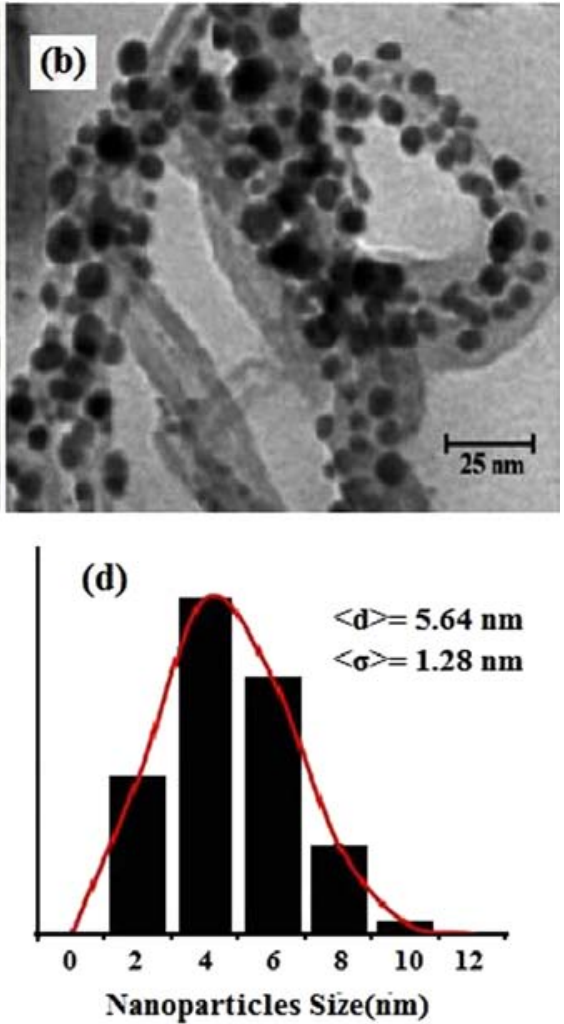

Fig. (8). (a) and (b) TEM images of FePt nanoparticles and FePt/CNT nanocomposite annealed in $600{ }^{\circ} \mathrm{C}$ for $2 \mathrm{~h}$, respectively, (c) and (d) histogram of FePt nanoparticles for them. 

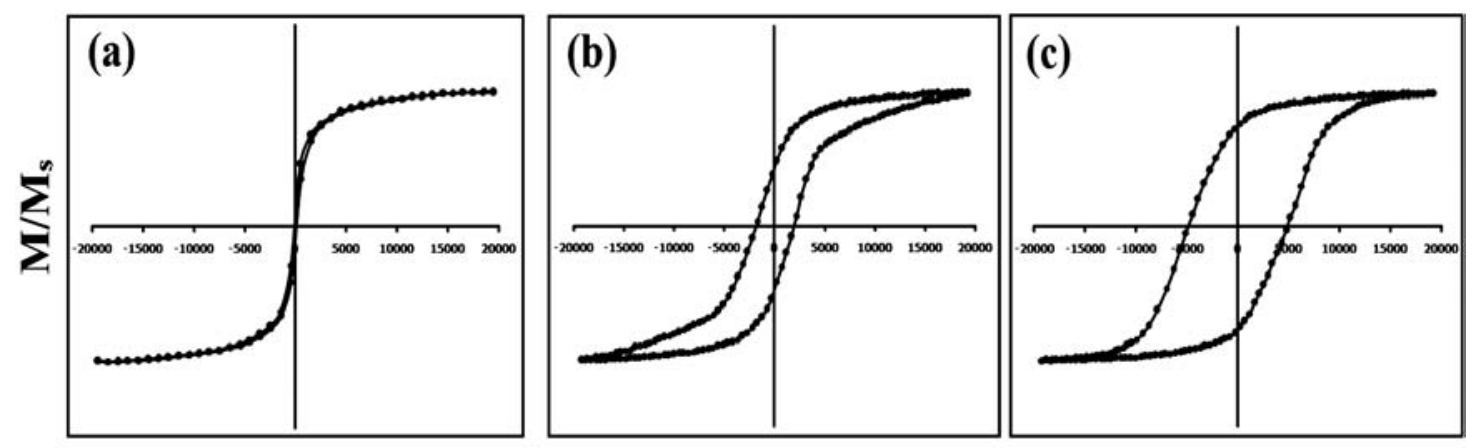

Field (G)

Fig. (9). Hysteresis loops of FePt/CNT composites: (a) as-synthesis, (b) after annealing in $600{ }^{\circ} \mathrm{C}$ for $2 \mathrm{~h}$ and (c) after annealing in $700{ }^{\circ} \mathrm{C}$ for $2 \mathrm{~h}$.

So the CNTs as an appropriate substrate prevent the nanoparticles from coalescence effectively. Histograms of FePt nanoparticles on the surface of nanotubes have been presented in Fig. (8c and d). According to histogram, the standard deviation of nanoparticle after annealing at $600^{\circ} \mathrm{C}$, is $\langle\sigma\rangle=0.68 \mathrm{~nm}$ that shows the almost uniform distribution of particles size. But, the nanoparticles size after annealing at $700^{\circ} \mathrm{C}$ is more non-uniform and larger.

Fig. (9) shows the hysteresis loops of FePt/CNTs nanocomposites at room temperature. The as-synthesis $\mathrm{FePt}$ nanoparticles have superparamagnetic behavior at room temperature for their low magnetic anisotropy.

Fig. (9b and c) presented the hysteresis loop of nanocomposites annealed at $600^{\circ} \mathrm{C}$ and $700^{\circ} \mathrm{C}$, respectively. The coercivity of $\mathrm{FePt} / \mathrm{CNT}$ composite at $600^{\circ} \mathrm{C}$ and $700^{\circ} \mathrm{C}$ is 2.2 $\mathrm{KOe}$ and $5.1 \mathrm{KOe}$, respectively, even though they have average size less than $6 \mathrm{~nm}$. With increasing in annealing temperature, the coercivity of nanoparticles increases significantly.

High coercivity after annealing process, like the XRD results, denotes that ordering of $\mathrm{L}_{0}$ phase improves at temperature higher than $600^{\circ} \mathrm{C}$.

\section{CONCLUSIONS}

In this paper, the finite size FePt nanoparticles with size of $2.5 \mathrm{~nm}$ were synthesized, without any surfactant agent. The as-synthesized FePt nanoparticles have chemically disordered fcc structure and they have superparamagnetic behavior because of their low magnetic anisotropy. Annealing at the temperature above $600^{\circ} \mathrm{C}$ leads to phase transition from disordered A1 phase to ordered L10 phase. The CNTs as a suitable substrate prevent the agglomeration of $\mathrm{FePt}$ nanoparticles during high thermal annealing (higher than $600^{\circ} \mathrm{C}$ ). The reaction between nanoparticles and the CNTs surfaces results in finite size nanoparticles with an average size of about $3.5 \mathrm{~nm}$ and $5.6 \mathrm{~nm}$ for annealing in $600^{\circ} \mathrm{C}$ and $700^{\circ} \mathrm{C}$, respectively and their magnetic coercivity reaches to $5.1 \mathrm{KOe}$ at $700^{\circ} \mathrm{C}$.

\section{CONFLICT OF INTEREST}

The authors confirm that this article content has no conflict of interest.

\section{ACKNOWLEDGEMENTS}

The authors would like to thank Mahdaneh Pardis company for financial support.

\section{REFERENCES}

[1] Sun, S.; Fullerton, E.E.; Weller, D.; Murray, C.B. Compositionally controlled FePt nanoparticle materials. IEEE Trans Magn., 2001, 37(4), 1239-1243.

[2] Nguyen, H.L.; Howard, L.E.M.; Giblin, S.R.; Tanner, B.K.; Terry, I.; Hughes, A.K.; Ross, I. M.; Serres, A.; Bu“rckstu"mmer, H.; Evans, J.S.O. Synthesis of Monodispersed fcc and fct FePt/FePd Nanoparticles by Microwave Irradiation. J. Mater. Chem., 2005, 15(48), 5136-5143.

[3] Chen, M.; Kim, J.; Liu, J.P.; Fan, H.; Sun, S. Synthesis of FePt Nanocubes and Their Oriented Self-Assembly. J. Am. Chem. Soc., 2006, 128(22), 7132-7133.

[4] Sun, S.; Murray, C.B.; Weller, D.; Folks, L.; Moser, A. Monodisperse $\mathrm{FePt}$ nanoparicles and ferromagnetic $\mathrm{FePt}$ nanocrystal superlattices. Science, 2000, 287(5460), 1989-1992.

[5] Rong, C.B.; Zhang, H.W.; Du, X.B.; Zhang, J.; Zhang, S.Y.; Shen, B.G. Investigation of hard magnetic properties of nanocomposite Fe-Pt magnets by micromagnetic simulation. J. Appl. Phys., 2004, 96(7), 3921-3924.

[6] Nandwana, V.; Elkins, K.E.; Poudyal, N.; Chaubey, G.S.; Yano, K.; Liu, J.P. Size and shape control of monodisperse FePt nanoparticles. J. Phys. Chem. C, 2007, 111(11), 4185-4189.

[7] Nakaya, M.; Kanehara, M.; Teranishi, T. One-pot synthesis of large FePt nanoparticles from metal salts and their thermal stability. Langmuir, 2006, 22(8), 3485-3487.

[8] Rong, C.B.; Nandwana, V.; Poudyal, N.; Chaubey, G.S.; Liu, J.P. Phase Transformation and Magnetic Hardening in Isolated FePt Nanoparticles. IEEE Trans Nanotech., 2009, 8(4), 437-443.

[9] Mito, M.; Komorida, Y.; Silva, N.J.O.; Tsuruda, H.; Deguchi, H.; Takagi, S.; Tajiri, T.; Iwamoto, T.; Kitamoto, Y. Particle-diameter dependence of the coercive field in FePt nanoparticles with a facecentered tetragonal structure. J. Appl. Phys., 2010, 108(12), 124315-124319.

[10] Zeng, H.; Yan, M.L.; Powers, N.; Sellmyer, D.J. Orientationcontrolled nonepitaxial L10 CoPt and FePt films. Appl. Phys. Lett., 2002, 80(13), 2350-2352.

[11] Kang, S.; Harrell, J.W.; Nikles, D.E. Reduction of the fcc to L10 ordering temperature for self-assembled $\mathrm{FePt}$ nanoparticles containing Ag. Nano Lett., 2002, 2(10), 1033-1036.

[12] Jia, Z.; Kang, S.; Shi, S.; Nikles, D.E.; Harrell, J.W. Size Effect on L10 Ordering and Magnetic Properties of Chemically Synthesized FePt and FePtAu Nanoparticles. J. Appl. Phys., 2005, 97(10), 10J310-312.

[13] Zeng, H.; Sabirianov, R.; Mryasov, O.; Yan, M. L.; Cho, K.; Sellmyer, D.J. Curie temperature of $\mathrm{FePt}: \mathrm{B}_{2} \mathrm{O}_{3}$ nanocomposite films. Phys. Rev. B, 2002, 66(18), 184425-6.

[14] Li, D.; Poudyal, N.; Nandwana, V.; Jin, Z.; Elkins, K.; Liu, J.P. Hard magnetic FePt nanoparticles by salt-matrix annealing. J. Appl. Phys., 2006, 99(8), 08E911-913. 
[15] Rong, C.B.; Li, D.; Nandwana, V.; Poudyal, N.; Ding, Y.; Wang, Z.L.; Zeng, H.; Liu, J.P. Size-dependent chemical and magnetic ordering in $\mathrm{L}_{0}$-FePt nanoparticles. Adv. Mat., 2006, 18(22), 29842988.

[16] Wellons, M.S.; Morris, W.H.; Gai, Z.; Shen, J.; Bently, J.; Witting, J.E.; Lukehart, C.M. Direct synthesis and size selection of ferromagnetic FePt nanoparticles. Chem. Mat., 2007, 19(10), 24832488.

[17] Yano, K.; Nandwana, V.; Poudyal, N.; Rong, C.B.; Liu, J.P. Rapid thermal annealing of FePt nanoparticles. J. Appl. Phys., 2008, 104(1), 013918-21.

[18] Tamada, Y.; Yamamoto, S.; Takano, M.; Nasu, S.; Ono, T. Wellordered $\mathrm{L} 1_{0}$-FePt nanoparticles synthesized by improved $\mathrm{SiO}_{2^{-}}$ nanoreactor method. Appl. Phys. Lett., 2007, 90(16), 162509162511.

[19] Varand, L.C.; Imaizumi, M.; Santos, F.J.; Jafelicci, M. Iron oxide versus $\mathrm{Fe} \mathrm{Pt} / \mathrm{Fe} \mathrm{O}$ : improved magnetic properties of core/shell nanoparticles for biomedical applications. IEEE Trans. Magn., 2008, 44(11), 4448-4451.

[20] Chaubey, G.S.; Nandwana, V.; Poudyal, N.; Rong, C.B.; Liu, J.P. Synthesis and characterization of bimagnetic bricklike nanoparticles. Chem. Mat., 2008, 20(2), 475-478.

Received: April 16, 2013

Revised: August 06, 2013

Accepted: August 29, 2013
[21] Li, Y.; Yang, R. Hydrogen storage in metal-organic frameworks by bridged hydrogen spillover. J. Am. Chem. Soc., 2006, 128(25), 8136-8137.

[22] Park, S.; Kim, B.; Lee, Y.; Cho, M. Influence of copper electroplating on high pressure hydrogen-storage behaviors of activated carbon fibers. Int. J. Hydrogen Energy, 2008, 33(6), 1706-1710.

[23] Peles, A.; Van de Walle, C. Role of charged defects and impurities in kinetics of hydrogen storage materials: a first principles study. Phys. Rev. B, 2007, 76(21), 4101-4106.

[24] Zacharia, R.; Kim, Y.; Kibria, A.; Nahm, K. Enhancement of hydrogen storage capacity of carbon nanotubes via spill-over from vanadium and palladium nanoparticles. Chem. Phys. Lett., 2005 , 412(4-6), 369-375.

[25] Osorio, A.G.; Silveira, I.C.L.; Bueno, V.L.; Bergmann, C.P. $\mathrm{H}_{2} \mathrm{SO}_{4} / \mathrm{HNO}_{3} / \mathrm{HCl}$-Functionalization and its effect on dispersion of carbon nanotubes in aqueous media. Appl. Surf. Sci., 2008, 255(5), 2485-2489.

[26] Robarts, B.W. X-ray measurement of order in CuAu. Acta Metall., 1954, 2(4), 597-603.

[27] Iwasaki, S.; Perpendicular magnetic recording - evolution and future. IEEE Trans. Magn., 1984, 20(5), 657-662. 